Pure and Applied Mathematics Quarterly

Volume 3, Number 4

(Special Issue: In honor of

Grisha Margulis, Part 1 of 2$)$

$1005-1026,2007$

\title{
Induced Representations of the Infinite Symmetric Group
}

\author{
N. V. Tsilevich* and A. M. Vershik*
}

\begin{abstract}
We study the representations of the infinite symmetric group induced from the identity representations of Young subgroups. It turns out that such induced representations can be either of type I or of type II. Each Young subgroup corresponds to a partition of the set of positive integers; depending on the sizes of blocks of this partition, we divide Young subgroups into two classes: large and small subgroups. The first class gives representations of type I, in particular, irreducible representations. The most part of Young subgroups of the second class give representations of type II and, in particular, von Neumann factors of type II. We present a number of various examples. The main problem is to find the so-called spectral measure of the induced representation. The complete solution of this problem is given for two-block Young subgroups and subgroups with infinitely many singletons and finitely many finite blocks of length greater than one.
\end{abstract}

\section{Contents}

1. Introduction 1006

2. Young subgroups and induced representations 1008

3. Induced representations of type I 1010

3.1. Large Young subgroups lead to type I representations 1010

3.2. Decomposition of type I representations ... 1011

3.3. Examples of type I induced representations 1012

4. Induced representations of type II 1016

Received May 3, 2006.

*Supported by the grants CRDF RUM1-2622-ST-04, INTAS 03-51-5018, RFBR 05-01-00899, and NSh-4329.2006.1. 
4.1. Small Young subgroups lead to representations of type II

4.2. Example: representations induced from a hook with ...

5. Examples of the spectral analysis of induced representations

5.1. Markov representations of the infinite symmetric group

5.2. The spectral analysis of two-block induced representations

5.3. Spectral measure of representations of type $\left(1^{\infty}, \nu\right)$

\section{INTRODUCTION}

In the classical representation theory of the symmetric groups, representations induced from Young subgroups (i.e., subgroups that leave some partition fixed) play a very prominent role (see, e.g., [4]). The irreducible components of such representations contain all irreducible representations, and in the classical approach, this allows one to establish a connection between Young diagrams and irreducible representations. Though at present there is an alternative approach to establishing this correspondence (avoiding induction from Young subgroups), nevertheless, the traditional problems concerning induction for representations of the infinite symmetric group are of independent importance. In this paper, we start the systematic study of representations of the infinite symmetric group $\mathfrak{S}_{\mathbb{N}}$ induced from infinite Young subgroups. Let us briefly present the main results.

One can roughly divide partitions and the corresponding Young subgroups into two classes: large partitions, which have finitely many finite blocks and an arbitrary number of infinite blocks (in this case, there is at least one infinite block), and small partitions, which have infinitely many finite blocks (without any assumption on the number of infinite blocks). In contrast to the case of finite symmetric groups, induction from the identity representations of a Young subgroup to $\mathfrak{S}_{\mathbb{N}}$ often gives an irreducible representation - this is the case for large Young subgroups corresponding to partitions with at most one finite block. This can be proved using the well-known Mackey criterion of irreducibility, or by direct arguments. Induction from an arbitrary large subgroup gives a type I representation, which, as is well known, has a unique decomposition into irreducible ones, and we describe this decomposition explicitly. It turns out to be finite, and, in general, its irreducible components are not induced representations.

In contrast to this case, induction from small Young subgroups gives type II representations. There is a well-developed theory of representations of $\mathfrak{S}_{\mathbb{N}}$ of type $\mathrm{II}_{1}$ with finite trace, but here we have another situation, which was not paid much 
attention to. Namely, the factor generated by the representation operators is of type $\mathrm{II}_{\infty}$, so that it has no finite trace, but its commutant is of type $\mathrm{II}_{1}$. Note that even in the case where the factor generated by the representation operators is of type $\mathrm{II}_{1}$, it may happen that it has no finite trace, because the cyclic vector of the representation is not cyclic for the commutant. In [15], such representations were called strange. The additional invariant of such representations is the so-called coupling constant.

It seems that the classification of such representations (up to equivalence) was not considered; it is not even known whether it is simpler than the classification of irreducible representations, which, as is well known, is a wild problem for groups that are not virtually commutative, such as $\mathfrak{S}_{\mathbb{N}}$. A very interesting problem is whether each irreducible representation of $\mathfrak{S}_{\mathbb{N}}$ appears in the decompositions of induced representations (more exactly, is weakly contained in induced representations), as is the case for the finite symmetric groups. It should be noted that there are few papers devoted to induced representations of the infinite symmetric group (see, e.g., papers by Binder [1, 2, 3], Obata [11, 12], and Hirai [6, 7]). In particular, [1] contains the irreducibility criterion for induction from Young subgroups (our Theorem 1(b)).

Models of representations of the infinite symmetric group $\mathfrak{S}_{\mathbb{N}}$ can be roughly divided into two classes (see [14]): substitutional models and spectral models. The first class contains models in which a group $G$ (not necessarily $\mathfrak{S}_{\mathbb{N}}$ ) acts by substitutions on some $G$-space. Induced (quasi-regular) representations belong to this type. The notion of a spectral model uses specific properties of the infinite symmetric group. The spectral analysis of a representation of a finite symmetric group means its decomposition into irreducible components indexed by Young diagrams (with some multiplicities). In a more rigorous style, this means that we diagonalize the image of the group algebra in the representation with respect to the so-called commutative Gelfand-Tsetlin algebra (see [20]). The group algebra of $\mathfrak{S}_{\mathbb{N}}$ also has a a distinguished maximal commutative subalgebra, the so-called Gelfand-Tsetlin algebra, whose spectrum is the space of infinite Young tableaux. The space of every cyclic unitary representation of $\mathfrak{S}_{\mathbb{N}}$ can be diagonalized with respect to this algebra. This gives a spectral model of this representation, which is realized in the Hilbert space $L_{\mu}^{2}(T, H)$, where $T$ is the space of infinite Young tableaux, $\mu$ is a measure on $T$ (the spectral measure of a cyclic vector), and $H$ is an auxiliary Hilbert space. Thus two problems arise, which are similar to problems of Fourier theory:

1) (Direct problem). To find a spectral model of a representation given in a substitutional realization.

2) (Inverse problem). To find a substitutional realization of a representation given in a spectral form. 
We consider these two problems as the main problems of this theory, and its main analytic and probabilistic component is the analysis of spectral measures; see, e.g., the authors' papers $[14,22,21]$. In this paper, we consider the first problem for some classes of induced representations. The first result in this direction, obtained in [22] and specified below, is that the representations induced from Young subgroups corresponding to partitions into two infinite blocks are simple and irreducible, and their spectral measures are Markov measures. Another nontrivial example considered in this paper is the class of induced representations corresponding to partitions with infinitely many singletons and finitely many finite blocks of length greater than one. In this case, the spectral measure is a convex combination of conditional Plancherel measures. A more precise spectral analysis of induced representations of $\mathfrak{S}_{\mathbb{N}}$ will be presented elsewhere.

The paper is organized as follows. In Sec. 2, we introduce necessary definitions and notation related to induced representations of $\mathfrak{S}_{\mathbb{N}}$ we are going to consider. Section 3 is devoted to induced representations of type I; namely, we give a condition under which the induced representation is of type I, describe the decomposition of such a representation into irreducible components, give an irreducibility criterion, and consider several classes of examples. In Sec. 4, in a similar way we deal with induced representations of type II: we give a condition under which the induced representation is of type II, describe the central decomposition of such a representation into factors, give a criterion of its being a factor, and consider several examples. Finally, in Sec. 5, we present two examples of the spectral analysis of the induced representations corresponding to partitions with two infinite blocks and to partitions with infinitely many singletons and finitely many finite blocks of length greater than one.

\section{Young SUbGRoups AND INDUCED REPRESENTATIONS}

We denote by $\mathfrak{S}_{n}$ the symmetric group of degree $n$.

The irreducible representations of the symmetric group $\mathfrak{S}_{n}$ are indexed by the set $\mathbb{Y}_{n}$ of Young diagrams with $n$ cells. Let $\pi_{\lambda}$ be the irreducible unitary representation of $\mathfrak{S}_{n}$ corresponding to a diagram $\lambda \in \mathbb{Y}_{n}$, and let $\operatorname{dim} \lambda$ be the dimension of $\pi_{\lambda}$. The branching of irreducible representations of the symmetric groups is described by the Young graph $\mathbb{Y}$. The set of vertices of the $\mathbb{Z}_{+}$-graded graph $\mathbb{Y}$ is $\cup_{n} \mathbb{Y}_{n}$, and two vertices $\mu \in \mathbb{Y}_{n-1}$ and $\lambda \in \mathbb{Y}_{n}$ are joined by an edge if and only if $\mu \subset \lambda$. By definition, the zero level $\mathbb{Y}_{0}$ consists of the empty diagram $\emptyset$.

Now let $\mathfrak{S}_{\mathbb{N}}=\cup_{n=1}^{\infty} \mathfrak{S}_{n}=\lim _{\mathfrak{S}} \mathfrak{S}_{n}$ be the infinite symmetric group with the fixed structure of an inductive limit of finite groups.

Consider an arbitrary partition $\Pi=\left(A_{1}, A_{2}, \ldots\right)$ of the set of positive integers $\mathbb{N}$ into disjoint subsets $A_{1}, A_{2}, \ldots$. The corresponding Young subgroup of the 
infinite symmetric group $\mathfrak{S}_{\mathbb{N}}$ is $\mathfrak{S}_{\Pi}=\mathfrak{S}_{A_{1}} \times \mathfrak{S}_{A_{2}} \times \ldots$, where $\mathfrak{S}_{A}$ is the group of all finite permutations of the elements of a set $A$.

Definition 1. Let $\Pi=\left(A_{1}, A_{2}, \ldots\right)$ be a partition of $\mathbb{N}$. For $i=1,2, \ldots, \infty$, denote by $k_{i} \in \mathbb{N} \cup\{\infty\}$ the number of $j$ such that $\left|A_{j}\right|=i$. We say that the partition $\Pi$ and the corresponding Young subgroup $\mathfrak{S}_{\Pi}$ are of type $\mathbf{k}=\left(\infty^{k_{\infty}}, 1^{k_{1}}, 2^{k_{2}}, \ldots\right)$.

It turns out that it is natural to divide partitions and the corresponding Young subgroups into two classes. Namely, we introduce the following definition.

Definition 2. A partition $\Pi$ of $\mathbb{N}$ and the corresponding Young subgroup $\mathfrak{S}_{\Pi}$ is called large if it has finitely many finite blocks (and, necessarily, at least one infinite block). Otherwise, i.e., if $\Pi$ has infinitely many finite blocks, it is called small.

Our purpose is to investigate the representations $I_{\Pi}=\operatorname{Ind}_{\mathfrak{S}_{\Pi}}^{\mathfrak{S}_{\mathbb{N}}} 1_{\mathfrak{S}_{\Pi}}$ of the infinite symmetric group $\mathfrak{S}_{\mathbb{N}}$ induced from the identity representations of Young subgroups.

Note that two Young subgroups $\mathfrak{S}_{\Pi_{1}}$ and $\mathfrak{S}_{\Pi_{2}}$ of the same type can be sent to each other by an automorphism of $\mathfrak{S}_{\mathbb{N}}$. If the partitions $\Pi_{1}$ and $\Pi_{2}$ are "tailequivalent" (i.e., can be obtained from each other by a finite permutation of $\mathbb{N}$ ) then this automorphism is an inner automorphism of $\mathfrak{S}_{\mathbb{N}}$, so that the subgroups $\mathfrak{S}_{\Pi_{1}}$ and $\mathfrak{S}_{\Pi_{2}}$ are conjugate in $\mathfrak{S}_{\mathbb{N}}$, and the representations $I_{\Pi_{1}}$ and $I_{\Pi_{2}}$ are obviously equivalent. In fact, the following lemma holds.

Lemma 1. The representations $I_{\Pi_{1}}$ and $I_{\Pi_{2}}$ are equivalent if and only if the Young subgroups $\mathfrak{S}_{\Pi_{1}}$ and $\mathfrak{S}_{\Pi_{2}}$ are conjugate in $\mathfrak{S}_{\mathbb{N}}$ (that is, the partitions $\Pi_{1}$ and $\Pi_{2}$ are tail-equivalent).

Proof. The "if" part is obvious. Let us prove the "only if" part. Assume that $I_{\Pi_{1}}$ and $I_{\Pi_{2}}$ are equivalent, and let $A$ be the corresponding intertwining operator. As in the proof of Theorem 1, one can show that this operator is determined by a function $\alpha$ defined on the left cosets $\mathfrak{S}_{\mathbb{N}} / \mathfrak{S}_{\Pi_{2}}$ and constant on the orbit of the left action of $\mathfrak{S}_{\Pi_{1}}$, that is, on left cosets lying in the same double coset $\mathfrak{S}_{\Pi_{1}} \backslash \mathfrak{S}_{\mathbb{N}} / \mathfrak{S}_{\Pi_{2}}$, so that $\alpha$ is concentrated on finite orbits, i.e., on double cosets that decompose into the union of finitely many left cosets. Then it is not difficult to show that there are no such finite orbits unless the partitions $\Pi_{1}$ and $\Pi_{2}$ differ by at most finitely many blocks, so that the lemma follows from the fact that for a finite symmetric group, the representations induced from the identity representations of two Young subgroups are equivalent if and only if these subgroups are conjugate.

Note that for irreducible induced representations, this assertion follows from a result of Mackey (see, e.g., [9, Corollary 3, p. 158]). 
We see that partitions of the same type $\mathbf{k}$ can lead to a continuum of nonequivalent induced representations. However, as we will see below, the rough properties of these representations (such as being irreducible or being a factor) depend only on the type $\mathbf{k}$.

Note that, in general, the properties of induced representations $\operatorname{Ind}_{H}^{G} 1_{H}$ are not continuous with respect to $H$. For example, consider the simplest case an increasing sequence of partitions of type $(\infty, n)$ (one infinite block and one finite block of size $n$ ). For a given $n$, all such Young subgroups are conjugate, so that the corresponding induced representations are equivalent. Hence the limits of sequences of invariants of induced representations also coincide. But such a sequence of partitions can converge to different types of partitions: in the limit, we have either the trivial partition into one block and hence the identity representation, or a partition with two infinite blocks, in which case the possible limits are a series of two-block representations, including a continuum of nonequivalent ones. At the same time, if we consider an increasing sequence of partitions with fixed cyclic vectors and embed them into one another preserving the cyclic vectors, then the matrix elements will be obviously continuous.

Thus, in what follows, we denote by $\xi$ the (normalized) distinguished cyclic vector of the representation $I_{\Pi}$. If we consider the realization of $I_{\Pi}$ in the $l^{2}$ space over the homogeneous space $\mathfrak{S}_{\mathbb{N}} / \mathfrak{S}_{\Pi}$, then $\xi$ is the delta function at the coset $\mathfrak{S}_{\Pi} \in \mathfrak{S}_{\mathbb{N}} / \mathfrak{S}_{\Pi}$. Let $\Pi_{n}=\Pi \cap\{1, \ldots, n\}$, denote by $I_{\Pi_{n}}$ the representation of the finite symmetric group $\mathfrak{S}_{n}$ induced from the identity representation of the Young subgroup $\mathfrak{S}_{\Pi_{n}} \subset \mathfrak{S}_{n}$, and let $\xi_{n}$ be the distinguished cyclic vector in $I_{\Pi_{n}}$. Then $I_{\Pi}$ is the inductive limit of $I_{\Pi_{n}}$, and the corresponding embeddings preserve the distinguished cyclic vectors. Note also that $I_{\Pi_{n}}$ can be identified with the restriction of $I_{\Pi}$ to $\mathfrak{S}_{n}$ in the cyclic hull $\mathfrak{S}_{n} \xi$ of $\xi$; then $\xi_{n}$ is identified with $\xi$.

\section{INDUCED REPRESENTATIONS OF TYPE I}

\subsection{Large Young subgroups lead to type I representations.}

Theorem 1. (a) The representation of the infinite symmetric group $\mathfrak{S}_{\mathbb{N}}$ induced from the identity representation of a large Young subgroup of type $\mathbf{k}=$ $\left(\infty^{k_{\infty}}, 1^{k_{1}}, 2^{k_{2}}, \ldots\right)$ with finitely many finite blocks (that is, with $\left.k_{1}+k_{2}+\ldots<\infty\right)$ is of type $I$ and decomposes into a finite sum of irreducible representations.

(b) The induced representation $I_{\Pi}$ of type $\left(\infty^{k_{\infty}}, 1^{k_{1}}, 2^{k_{2}}, \ldots\right)$ is irreducible if and only if the partition $\Pi$ contains at most one finite element, that is, $k_{1}+k_{2}+$ $\ldots \leq 1$.

Proof. (a) Let $\Pi=\left(A_{1}, A_{2}, \ldots\right)$ be a partition of type $\mathbf{k}$ with finitely many finite blocks. For convenience, assume that the blocks $A_{1}, \ldots, A_{n}$ of $\Pi$ are finite and the remaining blocks are infinite. For brevity, denote by $H=\mathfrak{S}_{\Pi}$ the corresponding 
Young subgroup, and let $\mathfrak{S}_{\text {fin }}=\mathfrak{S}_{\text {fin }}(\Pi)=\mathfrak{S}_{A_{1}} \times \ldots \times \mathfrak{S}_{A_{n}}$ be the finite Young subgroup corresponding to the finite blocks of $\Pi$.

By definition, the induced representation $I_{\Pi}$ acts in the space $l^{2}(X)$ of squaresummable functions on the (countable) homogeneous space $X=\mathfrak{S}_{\mathbb{N}} / H$. Denote by $R\left(I_{\Pi}\right)$ the space of intertwining operators for the representation $I_{\Pi}$, and let $A \in R\left(I_{\Pi}\right)$. The basis of $l^{2}(X)$ consists of the delta functions at the left cosets $s H \in X$ of $H$ in $\mathfrak{S}_{\mathbb{N}}$; by abuse of notation, we will denote a basis element by the same symbol as the corresponding coset. Then the operator $A$ is determined by a matrix $\left(a_{s H, t H}\right)_{s H, t H \in X}$ in this basis. Let $F \subset H \backslash G / H$ be the set consisting of those double cosets $\mathrm{HgH}$ that are unions of finitely many left cosets of $H$; and let $\operatorname{Comm}_{\mathfrak{S}_{\mathbb{N}}}(H)$ be the group, called the commensurator of $H$ in $\mathfrak{S}_{\mathbb{N}}$, consisting of $g \in \mathfrak{S}_{\mathbb{N}}$ such that $H g H \in F$. Denote by $\mathbf{1}_{x}$ the characteristic function of a double coset $x \in F$. Using the standard arguments, which go back to Mackey and his successors, one can show that the set of intertwining operators $R\left(I_{\Pi}\right)$ is generated by the operators $A_{x}, x \in F$, with matrix elements $a_{s H, t H}=\mathbf{1}_{x}\left(s^{-1} t H\right)$.

In our case, it is easy to see that $g \in \operatorname{Comm}_{H}^{\mathfrak{S}_{\mathbb{N}}}$ if and only if $g$ leaves invariant all infinite blocks of the partition $\Pi$, i.e., $g \in \mathfrak{S}_{\text {fin }}$. It follows that $R\left(I_{\Pi}\right)$ is isomorphic to the space $R\left(I_{\mathrm{fin}}\right)$ of intertwining operators for the representation $I_{\text {fin }}$ of the finite symmetric group $\mathfrak{S}_{N}$, where $N=k_{1}+k_{2}+\ldots$, induced from the identity representation of the Young subgroup $\mathfrak{S}_{\text {fin }}$. Denote by $P_{i} \in R\left(I_{\text {fin }}\right)$ the projections to the irreducible components of $I_{\mathrm{fin}}$. Then their images $\tilde{P}_{i}$ in $R\left(I_{\Pi}\right)$ determine the finite decomposition of $I_{\Pi}$ into irreducible components, and assertion (a) follows.

(b) It is easy to see that the condition $k_{1}+k_{2}+\ldots \leq 1$ is equivalent to $\operatorname{Comm}_{\mathfrak{S}_{\mathbb{N}}}(H)=H$, and the latter condition means that there are no nonscalar intertwining operators.

Remark. The claim (b) of this theorem was proved in [1]. It also easily follows from the irreducibility criterion for induced representations of discrete groups due to Mackey (see, e.g., [9, Corollary 2, p. 158]).

3.2. Decomposition of type I representations into irreducible components. Let $\mathfrak{S}_{\lambda}=\mathfrak{S}_{A_{1}} \times \ldots \times \mathfrak{S}_{A_{m}}$ be a Young subgroup of a finite symmetric group $\mathfrak{S}_{N}$ associated with a Young diagram $\lambda$ (which means that the lengths $\left|A_{k}\right|$ of blocks form the diagram $\lambda$ ). Then the decomposition of the representation of $\mathfrak{S}_{N}$ induced from the Young subgroup $\mathfrak{S}_{\lambda}$ into irreducible components is given by the following formula (see [4] and also [16]):

$$
\operatorname{Ind}_{\mathfrak{S}_{\lambda}}^{\mathfrak{S}_{N}} 1_{\mathfrak{S}_{\lambda}}=\sum_{\mu \unrhd \lambda} K_{\mu, \lambda} \pi_{\mu}
$$


where $\unrhd$ is the dominance ordering on partitions (see [8, Sec. I.1]) and $K_{\mu, \lambda}$ are the Kostka numbers (see [8, Sec. I.6]). (Recall that $\mu \unrhd \lambda$ if and only if $\mu_{1}+\mu_{2}+\ldots+\mu_{i} \geq \lambda_{1}+\lambda_{2}+\ldots+\lambda_{i}$ for all $i \geq 1$, and $K_{\mu, \lambda}$ is the number of semistandard Young tableaux of shape $\mu$ and weight $\lambda$.)

Combining these observations with the proof of Theorem 1, we obtain the following theorem.

Theorem 2. Let $\Pi$ be a partition of $\mathbb{N}$ with finitely many finite blocks. Denote by $\lambda=\lambda(\Pi)=1^{k_{1}} 2^{k_{2}} \ldots$ the Young diagram of size $N=k_{1}+k_{2}+\ldots$ formed by the lengths of the finite blocks of $\Pi$. Then

$$
I_{\Pi}=\sum_{|\mu|=N, \mu \unrhd \lambda} K_{\mu, \lambda} \pi_{\mu}^{k_{\infty}},
$$

where $\pi_{\mu}^{k_{\infty}}$ is the irreducible representation of $\mathfrak{S}_{\mathbb{N}}$ corresponding to the irreducible component $\pi_{\mu}$ of $I_{\mathrm{fin}}$ as described in the last paragraph of the proof of Theorem 1(a).

The representation $\pi_{\mu}^{k_{\infty}}$ can be explicitly described as follows. Let $m_{i}=\left|A_{i}\right|$. It is not difficult to see that the homogeneous space $X_{\Pi}=\mathfrak{S}_{\mathbb{N}} / \mathfrak{S}_{\Pi}$ can be realized as the space of ordered partitions of $\mathbb{N}$ into disjoint sets of given cardinalities

$$
r=\left(R_{1}, R_{2}, \ldots\right), \quad\left|R_{i}\right|=m_{i},
$$

tail-equivalent to the original partition $\Pi$ with the natural action of $\mathfrak{S}_{\mathbb{N}}$ by permutations. Moreover, the subset of all such partitions with fixed infinite blocks $R_{n+1}, R_{n+2}, \ldots$ can be identified with the homogeneous space $\mathfrak{S}_{N} / \mathfrak{S}_{\text {fin }}$, and the corresponding set of functions can be identified with the space of the representation $I_{\text {fin }}=\operatorname{Ind}_{\mathfrak{S}_{\text {fin }}}^{\mathfrak{S}_{N}} 1$. Let $P_{\mu}$ be the projection to an irreducible component $\pi_{\mu}$ of $I_{\text {fin }}$. Then the representation $\pi_{\mu}^{k_{\infty}}$ acts in the subspace obtained by applying the projection $P_{\mu}$ to the first "coordinates" $\left(R_{1}, \ldots, R_{n}\right)$ and leaving the other coordinates unchanged:

$$
\left(\tilde{P}_{\mu} f\right)\left(R_{1}, R_{2}, \ldots\right)=f\left(P_{\mu}\left(R_{1}, \ldots, R_{n}\right), R_{n+1}, \ldots\right) .
$$

\subsection{Examples of type $\mathbf{I}$ induced representations.}

3.3.1. Elementary representations. Let us consider induced representations of type $\mathbf{k}=(\infty, k)$ induced from Young subgroups with one infinite block and one finite block of size $n$. First of all, note that all Young subgroups of this type are conjugate, so that all representations of this type are equivalent; thus we may fix an arbitrary partition $\Pi$ of type $(\infty, k)$, say $\Pi=(\{1, \ldots, k\},\{k+1, k+2, \ldots\})$, and speak of the representation $I_{(\infty, k)}=I_{\Pi}$. By Theorem 1(b), this representation is irreducible. 
Recall the definition of an elementary representation of the infinite symmetric group $\mathfrak{S}_{\mathbb{N}}$.

Definition 3. A representation of the infinite symmetric group $\mathfrak{S}_{\mathbb{N}}$ is called elementary if it is the inductive limit of irreducible representations of finite symmetric groups $\mathfrak{S}_{n}$.

Remark 1. Thus an elementary representation is realized in the $l^{2}$ space on one class of tail-equivalent Young tableaux.

Remark 2. In this definition, we do not require that the embeddings preserve some sequence of fixed cyclic vectors. However, it is easy to see that the successive embeddings of the unit vector in the original one-dimensional representation of $\mathfrak{S}_{1}$ form such a sequence.

Obviously, the representation $I_{(\infty, k)}$ of $\mathfrak{S}_{\mathbb{N}}$ is the inductive limit of the representations $I_{n}$ of the finite symmetric groups $\mathfrak{S}_{n}$ induced from the identity representations of the Young subgroups $\mathfrak{S}_{\{1, \ldots, k\}} \times \mathfrak{S}_{\{k+1, \ldots, n\}}$. These representations are reducible; however, it turns out that only one irreducible component "survives" in the limit, so that the following proposition holds.

Proposition 1. The representation of the infinite symmetric group induced from a Young subgroup of type $(k, \infty)$ is elementary.

Proof. Observe that a two-row diagram $\lambda^{(n)}=(n-k, k)$ is majorized in the dominance ordering precisely by the diagrams $(n-m, m)$ with $m \leq k$, and all the corresponding Kostka numbers are equal to 1 . By (1), we have $I_{n}=$ $\sum_{m \leq k} \pi_{(n-m, m)}$. Consider the projection $P_{\lambda^{(n)}} \xi$ of the distinguished cyclic vector $\xi$ to the component $\pi_{\lambda^{(n)}}=\pi_{(n-k, k)}$. We have $\operatorname{dim} I_{n}=\frac{n !}{k !(n-k) !}, \operatorname{dim} \lambda^{(n)}=$ $\frac{n !(n-2 k+1)}{k !(n-k+1) !}$, and, obviously, $K_{\lambda^{(n)}, \lambda^{(n)}}=1$. Thus, by Lemma 2 (see Sec. 5 ),

$$
\left\|P_{\lambda^{(n)}} \xi\right\|^{2}=\frac{K_{\lambda^{(n)}, \lambda^{(n)}} \operatorname{dim} \lambda^{(n)}}{\operatorname{dim} I_{n}}=\frac{n-2 k+1}{n-k+1} \rightarrow 1 \quad \text { as } n \rightarrow \infty .
$$

It follows that the vectors $P_{\lambda^{(n)}} \xi$ converge to $\xi$, so that the inductive limit of the irreducible representations $\pi_{\lambda^{(n)}}$ coincides with $I_{\Pi}$, as required.

It is well known (see, e.g., $[10,14,22]$ and also Sec. 5.2) that the representation $I_{n}$ can be realized in the space of symmetric tensors of rank $k$ and dimension $n$. Then the representation $I_{(\infty, k)}$ can be realized in the space of infinite-dimensional symmetric tensors of rank $k$.

3.3.2. Representations of type $(\infty, \lambda)$. Let us consider induced representations of type $\mathbf{k}$ with one infinite block and finitely many finite blocks, i.e., $k_{\infty}=1$ and $k_{1}+k_{2}+\ldots=k<\infty$. Note that all representations of this type are equivalent. 
Proposition 2. Let $\Pi$ be a partition of $\mathbb{N}$ with one infinite block and finitely many finite blocks, and denote by $\lambda=\lambda(\Pi)=1^{k_{1}} 2^{k_{2}} \ldots$ the finite Young diagram of size $k=k_{1}+k_{2}+\ldots$ formed by the lengths of the finite blocks of $\Pi$. Then the decomposition if $I_{\Pi}$ into irreducible components is as follows:

$$
I_{\Pi}=\sum_{|\mu|=k, \mu \unrhd \lambda} K_{\mu, \lambda} \pi_{\mu}^{1},
$$

where $\pi_{\mu}^{1}$ is the elementary representation of $\mathfrak{S}_{\mathbb{N}}$, namely, the inductive limit $\pi_{\mu}^{1}=\lim _{n \rightarrow \infty} \pi_{\mu^{(n)}}$, where $\mu^{(n)}=\left(n-k, \mu_{1}, \mu_{2}, \ldots\right)$ is the diagram with first row $n-k$ and the other rows forming the diagram $\mu$.

Let us find the spectral measure of the distinguished cyclic vector $\xi$ in this case. The arguments generalize the proof of Proposition 1. Let $\lambda^{(n)}=\left(n-k, \lambda_{1}, \lambda_{2}, \ldots\right)$. Fix a digram $\mu=\left(\mu_{1}, \mu_{2}, \ldots\right) \unrhd \lambda,|\mu|=k$, set $\mu^{(n)}=\left(n-k, \mu_{1}, \mu_{2}, \ldots\right)$, and let $n \rightarrow \infty$. By Lemma 2,

$$
\left\|P_{\mu^{(n)}} \xi\right\|^{2}=\frac{K_{\mu^{(n)}, \lambda^{(n)}} \operatorname{dim} \mu^{(n)}}{\operatorname{dim} I_{n}},
$$

where $I_{n}=\operatorname{Ind}_{\mathfrak{S}_{\lambda}(n)}^{\mathfrak{S}_{n}} 1$. (Note that $I_{\Pi}$ is the inductive limit of $I_{n}$.) We have $\operatorname{dim} I_{n}=\frac{n !}{(n-k) ! \prod \lambda_{i} !} \sim \frac{n^{k}}{\prod \lambda_{i} !}$ as $n \rightarrow \infty$. Further, it follows from the hook length formula that $\operatorname{dim} \mu^{(n)} \sim \frac{n^{k} \operatorname{dim} \mu}{k !}$ as $n \rightarrow \infty$. Finally, it can easily be seen from the definition of Kostka numbers that $K_{\mu^{(n)}, \lambda^{(n)}}=K_{\mu, \lambda}$ (indeed, $K_{\mu^{(n)}, \lambda^{(n)}}$ is the number of fillings of the shape $\mu^{(n)}$ with $n-k$ zeros, $\lambda_{1}$ ones, etc.; but, obviously, the $n-k$ zeros must occupy exactly the first row of $\left.\mu^{(n)}\right)$. Thus we obtain

$$
\lim _{n \rightarrow \infty}\left\|P_{\mu^{(n)}} \xi\right\|^{2}=\frac{K_{\mu, \lambda} \operatorname{dim} \mu \prod \lambda_{i} !}{k !} .
$$

Note that the sum of the right-hand sides over all $\mu \unrhd \lambda$ is equal to 1 . It follows that the spectral measure of $\xi$ is discrete and supported by a finite set of classes of tail-equivalent tableaux indexed by diagrams $\mu \unrhd \lambda$. In particular, $I_{\Pi}$ is a finite sum of elementary representations.

Remark 1. Thus we see that all elementary representations of $\mathfrak{S}_{\mathbb{N}}$ corresponding to inductive limits of sequences of irreducible representations associated with Young diagrams with growing first row can be obtained by induction from Young subgroups.

Remark 2. Note that for $k_{\infty} \geq 2$, the irreducible representation $\pi_{\mu}^{k_{\infty}}$ of $\mathfrak{S}_{\mathbb{N}}$ is no longer elementary.

Important particular case: a hook with infinite hand and finite leg. Let us consider the representation induced from "a hook with infinite hand and finite leg," i.e., a Young subgroup with one infinite block and $n$ one-point blocks 
(the case of a hook with infinite hand and infinite leg will be considered in Sec. 4.2, and that of a hook with finite hand and infinite leg, in Sec. 5.3). In this case, $\lambda(\Pi)$ is the column diagram $1^{n}$ and $\mathfrak{S}_{\text {fin }}=\{e\}$, so that $I_{\text {fin }}$ is the regular representation $\operatorname{Reg}_{n}$ of $\mathfrak{S}_{n}$. Thus the decomposition (1) turns into the well-known formula

$$
\operatorname{Reg}_{n}=\sum_{|\mu|=n} \operatorname{dim} \mu \cdot \pi_{\mu}
$$

and the decomposition (4) takes the form

$$
I_{\left(\infty, 1^{n}\right)}=\sum_{|\mu|=n} \operatorname{dim} \mu \cdot \pi_{\mu}^{1} .
$$

In this case, the homogeneous space $X_{\Pi}$ can be identified (by "forgetting" the infinite part of the partition $r$ in $(3))$ with the space of $n$-sequences $\left(r_{1}, \ldots, r_{n}\right)$ of distinct positive integers, that is, $l^{2}\left(X_{\Pi}\right)$ can be identified with the space of diagonal-free infinite-dimensional tensors of rank $n$. On the other hand, the regular representation $I_{\text {fin }}=\operatorname{Reg}_{n}$ has a realization in the space of tensors of dimension $n$. The projections $P_{\mu}$ to the irreducible components of $I_{\text {fin }}$ are the standard Young symmetrizers; and the projections to the primary components, i.e., subspaces of tensors with given symmetry type, are the central Young symmetrizers (see, e.g., [5, 23]). Applying these symmetrizers to infinite-dimensional tensors of rank $n$, we obtain the irreducible (or primary) components of $I_{\left(\infty, 1^{n}\right)}$.

For example, the representation $I_{\left(\infty, 1^{2}\right)}$ acts in the space of infinite-dimensional tensors of rank 2 , and

$$
I_{\left(\infty, 1^{2}\right)}=\pi_{(2)}^{1}+\pi_{\left(1^{2}\right)}^{1},
$$

where the projections to the irreducible components are given by symmetrizing and antisymmetrizing:

$$
P_{(2)} T_{r_{1}, r_{2}}=\frac{1}{2}\left(T_{r_{1}, r_{2}}+T_{r_{2}, r_{1}}\right), \quad P_{\left(1^{2}\right)} T_{r_{1}, r_{2}}=\frac{1}{2}\left(T_{r_{1}, r_{2}}-T_{r_{2}, r_{1}}\right) .
$$

As follows from (5), the spectral measure of the distinguished cyclic vector $\xi$ in this case has the Plancherel weights (see Sec. 5.1):

$$
\lim _{n \rightarrow \infty}\left\|P_{\mu^{(n)}} \xi\right\|^{2}=\frac{\operatorname{dim}^{2} \mu}{k !} .
$$

3.3.3. Two-block representations. By Theorem 1(b), induced representations of type $\infty^{2}$, induced from Young subgroups with two infinite blocks, are irreducible. (Note that there is a continuum of nonequivalent representations of this type.) As mentioned above, in the case of a two-row diagram $\lambda$, all Kostka numbers $K_{\mu, \lambda}$ for $\mu \unrhd \lambda$ are equal to 1 . Thus we obtain the following assertion.

Proposition 3. The representation of the infinite symmetric group induced from a two-block Young subgroup is irreducible, and its spectrum with respect to the Gelfand-Tsetlin algebra is simple. 
As shown in [22], in this case one can obtain a complete spectral analysis of the induced representations. We present these details in Sec. 5.2.

\section{INDUCED REPRESENTATIONS OF TYPE II}

4.1. Small Young subgroups lead to representations of type II. Now let us consider small Young subgroups $\mathfrak{S}_{\Pi}$. We will assume that the partition $\Pi$ has finitely many finite blocks of finite multiplicities and denote by $\nu=\nu(\Pi)$ the Young diagram formed by the sizes of these blocks.

Theorem 3. Let $\Pi$ be a partition of $\mathbb{N}$ of type $\mathbf{k}=\left(\infty^{k_{\infty}}, 1^{k_{1}}, 2^{k_{2}}, \ldots\right)$ that has finitely many finite blocks of finite multiplicities (i.e., $\sum_{j<\infty: k_{j}<\infty} k_{j}<\infty$ ), and assume that there exists at least one $i \in \mathbb{N}$ with $k_{i}=\infty$. Then

(a) The representation $I_{\Pi}$ of the infinite symmetric group $\mathfrak{S}_{\mathbb{N}}$ is of type II.

(b) This representation is a (type II) factor representation if and only if the diagram $\nu(\Pi)$ consists of at most one row.

(c) (Central decomposition) Let $N=|\nu(\Pi)|$. The representation $I_{\Pi}$ is a finite sum of type II factor representations indexed by the primary components of the representation $\operatorname{Ind}_{\mathfrak{S}_{\nu(\Pi)}}^{\mathfrak{S}_{N}} 1$, i.e., by Young diagrams $\mu$ such that $|\mu|=N$ and $\mu \unrhd$ $\nu(\Pi)$.

Proof. Let $J=J_{\Pi}=\left\{j \in \mathbb{N}: k_{j}=\infty\right\}$. By our assumptions, $J \neq \emptyset$. For each $j \in J$, let $B_{1}^{(j)}, B_{2}^{(j)}, \ldots$ be all blocks of size $j$, and set $\mathfrak{S}^{(j)}=\mathfrak{S}_{B_{1}^{(j)}} \times \mathfrak{S}_{B_{2}^{(j)}} \times \ldots$ In a similar way, let $A_{1}, \ldots, A_{n}$ be all finite blocks of finite multiplicities, and let $\mathfrak{S}_{\nu}=\mathfrak{S}_{\nu(\Pi)}=\mathfrak{S}_{A_{1}} \times \mathfrak{S}_{A_{2}} \times \ldots$ be the corresponding finite Young subgroup of $\mathfrak{S}_{N}$, where $N=\left|A_{1}\right|+\left|A_{2}\right|+\ldots=|\nu(\Pi)|$. We also denote $I_{\nu}=\operatorname{Ind}_{\mathfrak{S}_{\nu}}^{\mathfrak{S}_{N}} 1$.

It follows from the proof of Theorem 1 that the set of intertwining operators for $I_{\Pi}$ (i.e., the commutant $\mathfrak{A}^{\prime}$ of the algebra $\mathfrak{A}$ generated by the representation operators) decomposes into the tensor product

$$
\mathfrak{A}^{\prime}=R\left(I_{\nu}\right) \otimes \bigotimes_{j \in J} R_{j},
$$

where $R\left(I_{\nu}\right)$ is the set of intertwining operators for $I_{\nu}$ and $R_{j}$ is the algebra of operators generated by finite permutations of the sets $B_{1}^{(j)}, B_{2}^{(j)}, \ldots$. Obviously, $R_{j}$ is (algebraically) isomorphic to the algebra generated by the regular representation of the infinite symmetric group, which is a factor of type $\mathrm{II}_{1}$. Thus $\otimes \bigotimes_{j \in J} R_{j}$ is a factor of type II. Now if $\nu$ consists of a single row, than the representation $I_{\nu}$ is irreducible and $R\left(I_{\nu}\right)$ consists of scalar operators, so that the whole $\mathfrak{A}^{\prime}$ is a factor of type II. Otherwise, taking in $R\left(I_{\nu}\right)$ the projections to the 
primary components of $I_{\nu}$, we obtain the central decomposition of $I_{\Pi}$ into a sum of factors.

Remark. Assume that $\# J=\#\left\{j \in \mathbb{N}: k_{j}=\infty\right\}<\infty$ and $\nu(\Pi)$ consists of at most one row, i.e., $I_{\Pi}$ is a factor representation. Then the commutant $\mathfrak{A}^{\prime}=\otimes_{j \in J} R_{j}$ is a finite tensor product of factors of type $\mathrm{II}_{1}$, which is a factor of type $\mathrm{II}_{1}$. At the same time, it is not difficult to see that if $\Pi$ is not the trivial partition into singletons (i.e., $I_{\Pi}$ is not the regular representation), then the algebra $\mathfrak{A}$ itself is a factor of type $\mathrm{II}_{\infty}$.

Theorems 1 and 3, which describe induced representations of type I and II, respectively, do not exhaust all induced representations. Namely, they leave out the case when the partition $\Pi$ has infinitely many finite blocks of finite multiplicities, i.e., $\#\left\{i \in \mathbb{N}: k_{i}<\infty\right\}=\infty$. The most important example of such a partition is a partition that has no infinite blocks and at most one block of each finite size $\left(k_{\infty}=0, k_{i} \leq 1\right.$ for all $\left.i \in \mathbb{N}\right)$. It is natural to conjecture that the corresponding representation is also of type II.

4.2. Example: representations induced from a hook with infinite hand and infinite leg. Let us consider in more detail the induced representation of type $\left(\infty, 1^{\infty}\right)$ associated with a partition $\Pi$ having one infinite block and infinitely many singletons. Note that there is a continuum of nonequivalent representations of this type.

In this case, the homogeneous space $X$ is the space of "infinite-dimensional tensors of infinite rank," i.e., infinite sequences $\left(i_{1}, i_{2}, \ldots\right)$ of positive integers. It can be also described as follows. Let $\mathbb{N}_{0}$ be the subset of $\mathbb{N}$ consisting of all singletons of $\Pi$. Then the space $l^{2}(X)$ can be identified with the space of summable injections $f: \mathbb{N}_{0} \rightarrow \mathbb{N}$. The representation $I=I_{\Pi}$ is generated by the left action of $\mathfrak{S}_{\mathbb{N}}$ by $I_{g} f(n)=g^{-1} f(n)$. Denote by $\mathfrak{A}$ the von Neumann algebra generated by this representation. It is easy to see that the commutant $\mathfrak{A}^{\prime}$ of this algebra, i.e., the set of intertwining operators, is generated by the right action of the group $\mathfrak{S}\left(\mathbb{N}_{0}\right)$ of finite permutations of $\mathbb{N}_{0}$ by substitutions: $T_{\sigma} f(n)=$ $f\left(\sigma^{-1} n\right)$. In particular, $\mathfrak{A}^{\prime}$ is algebraically isomorphic to the von Neumann algebra generated by the regular representation of the infinite symmetric group, i.e., is a factor of type $\mathrm{II}_{1}$. As to the factor $\mathfrak{A}$ itself, it is easy to see that it decomposes into the infinite direct sum of factors of type $\mathrm{II}_{1}$. Indeed, for each infinite subset $B \subset \mathbb{N}$, let $H_{B}$ be the subspace of $l^{2}(X)$ consisting of functions $f$ such that $f^{-1}\left(\mathbb{N}_{0}\right)=B$. This subspace is obviously invariant with respect to $\mathfrak{A}^{\prime}$, so that $l^{2}(X)=\oplus_{B} H_{B}$ is the desired decomposition of $\mathfrak{A}$ into the infinite direct sum of factors of type $\mathrm{II}_{1}$. Thus we obtain the following proposition. 
Proposition 4. The induced representation of type $\left(\infty, 1^{\infty}\right)$ of the infinite symmetric group $\mathfrak{S}_{\mathbb{N}}$ is a factor representation. The von Neumann algebra $\mathfrak{A}$ generated by the representation operators is a factor of type $I I_{\infty}$, a direct sum of isomorphic factors of type $I I_{1}$. Its commutant $\mathfrak{A}^{\prime}$ is a factor of type $I I_{1}$ algebraically isomorphic to the factor generated by the regular representation of $\mathfrak{S}_{\mathbb{N}}$.

Note that the distinguished cyclic vector $\xi$, which in this realization is just the identical function $\xi(n) \equiv n$, is a cyclic vector for $\mathfrak{A}$, but not for $\mathfrak{A}^{\prime}$. Thus it defines a finite trace on the commutant by the formula $\operatorname{tr}(A)=(A \xi, \xi), A \in \mathfrak{A}^{\prime}$ (which is just the trace corresponding to the regular representation of the group $\mathfrak{S}\left(\mathbb{N}_{0}\right)$, i.e., coincides on the elements of $\mathfrak{S}\left(\mathbb{N}_{0}\right)$ with the delta function at the identity element), but there is no finite trace on $\mathfrak{A}$.

Remark. The described construction is a particular case of the following one. We have the standard left action of a group $G$ on the discrete homogeneous space $X=G / H$, where $H$ is a subgroup of $G$, and the corresponding unitary representation of $G$ in $l^{2}(X)$. The commutant of the corresponding algebra is generated by the automorphisms of the $G$-space $X$, i.e., by the right action of the group $N(H) / H$, where $N(H)$ is the normalizer of $H$ in $G$. The specific property of this situation is that both left and right representations are generated by substitutional actions of $G$ and $N(H) / H$, respectively (cf. [15]).

\section{EXAmples of the SPECTRAL ANALYSis of induCED REPRESENTATIONS}

In this section, we will consider two nontrivial examples of classes of induced representations for which we can explicitly find the spectral measure of the distinguished cyclic vector and, consequently, obtain a spectral realization.

To this end, the following simple lemma is useful. Consider the restriction $I_{n}$ of $I_{\Pi}$ to a finite symmetric group $\mathfrak{S}_{n}$ in the cyclic hull $\mathfrak{S}_{n} \xi$ of the distinguished cyclic vector $\xi$ (which is the representation induced from the identity representation of the Young subgroup $H=\mathfrak{S}_{\Pi_{n}}$ of $\mathfrak{S}_{n}$ associated with the partition $\Pi_{n}=$ $\Pi \cap\{1, \ldots, n\})$. Let $\lambda$ be a Young diagram with $n$ cells, and denote by $P_{\lambda}$ the projection from $I_{n}$ to the primary component $V_{\lambda}$ that is a multiple of $\pi_{\lambda}$.

Lemma 2. The squared norm $\left\|P_{\lambda} \xi\right\|^{2}$ is equal to the relative dimension of $V_{\lambda}$ in $I_{n}$, i.e.,

$$
\left\|P_{\lambda} \xi\right\|^{2}=\frac{\operatorname{dim} V_{\lambda}}{\operatorname{dim} I_{n}} .
$$

Proof. It is well known (see, e.g., [13]) that the projection $P_{\lambda}$ is given by the formula

$$
P_{\lambda}=\frac{\operatorname{dim} \lambda}{n !} \sum_{g \in \mathfrak{S}_{n}} \chi_{\lambda}(g) I_{n}(g),
$$


where $\chi_{\lambda}$ is the character of $\pi_{\lambda}$. Then

$$
\left(P_{\lambda} \xi, P_{\lambda} \xi\right)=\left(P_{\lambda} \xi, \xi\right)=\frac{\operatorname{dim} \lambda}{n !} \sum_{g \in \mathfrak{S}_{n}} \chi_{\lambda}(g)\left(I_{n}(g) \xi, \xi\right)=\frac{\operatorname{dim} \lambda}{n !} \sum_{h \in H} \chi_{\lambda}(h),
$$

because $\left(I_{n}(g) \xi, \xi\right)$ is equal to 1 if $g \in H$ and 0 otherwise. But the latter sum is equal to $\left(\left.\chi_{\lambda}\right|_{H}, 1_{H}\right) \cdot|H|$, which is in turn equal to $\left(\chi_{\lambda}, I_{n}\right) \cdot|H|$ by the Frobenius reciprocity, so that we obtain

$$
\left\|P_{\lambda} \xi\right\|^{2}=\frac{\left(\chi_{\lambda}, I_{n}\right) \operatorname{dim} \lambda \cdot|H|}{n !}=\frac{\operatorname{dim} V_{\lambda}}{\operatorname{dim} I_{n}}
$$

because $\left(\chi_{\lambda}, I_{n}\right)$ is the multiplicity of $\pi_{\lambda}$ in $I_{n}$ and $\operatorname{dim} I_{n}=\frac{n !}{|H|}$.

5.1. Markov representations of the infinite symmetric group. In this section, we recall necessary notions from the representation theory of the symmetric groups and the notions of simple and Markov representations.

Denote by $T_{\lambda}$ the set (consisting of $\operatorname{dim} \lambda$ elements) of Young tableaux of shape $\lambda \in \mathbb{Y}_{n}$, or, which is the same, the set of paths in the Young graph from the empty diagram $\emptyset$ to $\lambda$. Let $T_{n}=\cup_{\lambda \in \mathbb{Y}_{n}} T_{\lambda}$ be the set of Young tableaux with $n$ cells. According to the branching rule for irreducible representations of the symmetric groups, the space $V_{\lambda}$ of the irreducible representation $\pi_{\lambda}$ decomposes into the sum of one-dimensional subspaces indexed by the tableaux $u \in T_{\lambda}$. The basis $\left\{h_{u}\right\}_{u \in T_{\lambda}}$ consisting of vectors of these subspaces is called the Gelfand-Tsetlin basis. It is an eigenbasis for the Gelfand-Tsetlin algebra $\mathrm{GZ}_{n}$, the subalgebra in the group algebra $\mathbb{C}\left[\mathfrak{S}_{n}\right]$ generated by the centers $Z\left[\mathfrak{S}_{1}\right], Z\left[\mathfrak{S}_{2}\right], \ldots, Z\left[\mathfrak{S}_{n}\right]$ (see [20]).

Denote by $T=\lim T_{n}$ the space of infinite Young tableaux (the projective limit of $T_{n}$ with respect to the natural projections forgetting the tail of a path). With the topology of coordinatewise convergence $T$ is a totally disconnected metrizable compact space. The tail equivalence relation $\sim$ on $T$ is defined as follows: paths $s=\left(s_{1}, s_{2}, \ldots\right)$ and $t=\left(t_{1}, t_{2}, \ldots\right)$ are equivalent if and only if $s_{k}=t_{k}$ for all sufficiently large $k$. Denote by $[t]_{n} \in T_{n}$ the initial segment of length $n$ of a tableau $t \in T$. Given a finite tableau $u \in T_{n}$, denote by $C_{u}=\left\{t:[t]_{n}=u\right\}$ the corresponding cylinder set; for $\lambda \in \mathbb{Y}_{n}$, let $C_{\lambda}=\left\{t: t_{n}=\lambda\right\}=\cup_{u \in T_{\lambda}} C_{u}$ be the set of all paths passing through $\lambda$.

Definition 4. A measure $M$ on the space $T$ is called Markov if for every $n \in \mathbb{N}$ the following condition holds: for any diagrams $\lambda \in \mathbb{Y}_{n}$ and $\Lambda \in \mathbb{Y}_{n+1}$ such that $\Lambda \subset \lambda$ and for any path $u \in T_{\lambda}$, the events $C_{u}$ ("the past") and $C_{\Lambda}$ ("the future") are independent given $C_{\lambda}$ ("the present").

In other words, a random tableau $t=\left(t_{1}, t_{2}, \ldots\right)$, regarded as a sequence of random variables $t_{n}$, where $t_{n}$ takes values in the set $\mathbb{Y}_{n}$ of Young diagrams with $n$ 
cells, is a Markov chain in the ordinary sense. In terms of transition probabilities, this means that the transition probability $\frac{M\left(C_{\Lambda} \cap C_{u}\right)}{M\left(C_{u}\right)}$ depends only on the form $\lambda$ of a tableau $u$, but not on the tableau itself. Note that the "forward" and "backward" Markov properties are equivalent, so that the definition of a Markov measure can be formulated in a similar way in terms of cotransition probabilities.

One of the most important examples of Markov measures on $T$ is the Plancherel measure $P$, which is the spectral measure of the regular representation of $\mathfrak{S}_{\mathbb{N}}$.

Definition 5. The Plancherel measure on the space T of infinite Young tableaux is the Markov measure with transition probabilities $\operatorname{Prob}(\lambda, \Lambda)=\frac{\operatorname{dim} \Lambda}{(n+1) \operatorname{dim} \lambda}$, where $\lambda \in \mathbb{Y}_{n}, \Lambda \in \mathbb{Y}_{n+1}, \lambda \subset \Lambda$.

The cylinder distributions $P_{n}$ of the Plancherel measure are given by the formula $P_{n}\left(C_{\lambda}\right)=\frac{\operatorname{dim}^{2} \lambda}{n !}, \lambda \in \mathbb{Y}_{n}$. This distribution on the set of Young diagrams with $n$ cells is called the Plancherel measure on Young diagrams (see [17]).

Now assume that we have a cyclic representation $\pi$ of the group $\mathfrak{S}_{n}$ in a space $V$ that has a simple spectrum (i.e., decomposes into the sum of pairwise nonequivalent irreducible representations) and a unit cyclic vector $\xi$ in this representation.

Definition 6. We say that $\xi$ is a Markov vector if its spectral measure with respect to the Gelfand-Tsetlin algebra is Markov.

Lemma 3. Let $\pi$ be a unitary representation of the group $\mathfrak{S}_{n}$ with simple spectrum. A cyclic vector $\xi$ of the representation $\pi$ is Markov if and only if for every $k<n$, the representation of the group $\mathfrak{S}_{k}$ in the cyclic hull $\mathfrak{S}_{k} \xi$ of $\xi$ with respect to $\mathfrak{S}_{k}$ has a simple spectrum.

Proof. Clearly, a cyclic vector is Markov if for every $k<n$ and every diagram $\lambda \in \mathbb{Y}_{k}$, the probability of any tableau with this diagram does not depend on the continuation of this tableau to the level $n$. In terms of representations and cyclic vectors, this means that the norm of the projection of the cyclic vector to the subspace of the representation of the group $\mathfrak{S}_{k}$ equivalent to $\pi_{\lambda}$ does not depend on the way in which we have arrived at this subspace.

Now we use the following simple lemma.

Lemma 4. Assume that in a finite-dimensional Hilbert space $H$ there is a unitary representation of a group $G$ that is primary, i.e., decomposes into the direct (not necessarily orthogonal) sum $H=H_{1} \oplus H_{2} \oplus \ldots \oplus H_{n}$ of equivalent irreducible representations, and in each of them there is a cyclic vector $v_{i} \in H_{i}, i=1, \ldots, n$. Then the following two assertions are equivalent:

1. For any $i, j$, there exists an isometry $T_{i, j}: H_{i} \rightarrow H_{j}$ intertwining the corresponding representations such that $T_{i, j} v_{i}=v_{j}$.

2. In the cyclic hull of the vector $v=\sum v_{i}$, the representation is irreducible. 
Proof. Without loss of generality assume that $n=2$. Then the representation in the cyclic hull of $v$ is irreducible if and only if this vector is of rank 1, i.e., $v$, regarded as an element of the tensor product, has the form $x \otimes x$.

Now let $\xi_{\mu}$ be the projection of $\xi$ to the irreducible component $\pi_{\mu}$ of $\pi$. Fix $k<n$ and an irreducible representation $\pi_{\lambda}$ of $\mathfrak{S}_{k}$. Consider the restriction of $\pi$ to $\mathfrak{S}_{k}$, and let $H=H_{1} \oplus H_{2} \oplus \ldots \oplus H_{n}$, where $H_{i} \simeq \pi_{\lambda}$, be the primary component of this restriction corresponding to $\pi_{\lambda}$. The Markov property means that the norms of the projections of $\xi_{\mu}$ to $H_{i}$ coincide, which is equivalent, in view of Lemma 4, to the fact that $\pi_{\lambda}$ has multiplicity 1 in the decomposition of the representation of $\mathfrak{S}_{k}$ in the cyclic hull $\mathfrak{S}_{k} \xi$ of $\xi$.

Now let us consider representations of the infinite symmetric group $\mathfrak{S}_{\mathbb{N}}$.

If we are given a quasi-invariant measure $\mu$ on the space of Young tableaux $T$ and a 1-cocycle $c$ on pairs of tail-equivalent paths taking values in the group of complex numbers of modulus 1 , then we can construct a unitary representation of the group $\mathfrak{S}_{\mathbb{N}}$ in the space $L^{2}(T, \mu)$ as follows (see, e.g., [18]). Recall that the Fourier transform allows one to realize the group algebra $\mathbb{C}\left[\mathfrak{S}_{\mathbb{N}}\right]$ of the infinite symmetric group as the cross product constructed from the commutative algebra of functions on the space of tableaux $T$ (Gelfand-Tsetlin algebra) and the tail equivalence relation. The desired representation is given by

$$
L_{g} h(s)=\sum_{t \sim s} \sqrt{\frac{d \mu(s)}{d \mu(t)}} \hat{g}(s, t) c(s, t) h(t), \quad h \in L^{2}(T, \mu),
$$

where $\hat{g}$ is the function on pairs of tail-equivalent paths corresponding to an element $g \in \mathfrak{S}_{\mathbb{N}}$ (the Fourier transform of $g$ ). Note that the cocycle is trivial on the space of finite tableaux.

Definition 7. A representation of the infinite symmetric group $\mathfrak{S}_{\mathbb{N}}$ is called simple if it is the inductive limit of representations of the finite symmetric groups $\mathfrak{S}_{n}$ with simple spectrum.

Definition 8. A representation $\pi$ of the group $\mathfrak{S}_{\mathbb{N}}$ with simple spectrum is called Markov if the space of $\pi$ contains a cyclic vector whose spectral measure (with respect to the Gelfand-Tsetlin algebra) is Markov.

Note that a representation with simple spectrum is Markov if and only if the measure $\mu$ in its realization (8) is Markov.

The following theorem is an easy consequence of Lemma 3.

Theorem 4 ([22]). A representation of the infinite symmetric group is Markov if and only if it is simple. 
5.2. The spectral analysis of two-block induced representations. In this section, we present the complete spectral analysis of induced representations of type $\infty^{2}$. By Theorem 1, these representations are irreducible. It turns out that in this case the spectral measure of the distinguished cyclic vector is a Markov measure on the space of infinite Young tableaux $T$. The material of this section is mostly borrowed from the authors' paper [22]. Theorem 5 is proved there using the so-called tensor model of two-row representations of the symmetric groups (i.e., representations induced from two-block Young subgroups). See [22] for a detailed description of this model (in particular, explicit formulas for the Gelfand-Tsetlin basis).

A partition $\mathbb{N}=A \cup B$ of type $\infty^{2}$ is uniquely determined by an infinite sequence $\xi=\xi_{1} \xi_{2} \ldots$ of 0 's and 1's (an "infinite tensor"), where $\xi_{i}=1$ if $i \in A$, and $\xi_{i}=0$ if $i \in B$. Then the induced representation in question is equivalent to the natural substitutional representation of $\mathfrak{S}_{\mathbb{N}}$ on infinite sequences in the cyclic hull of the sequence $\xi$, which we will denote by $\pi_{\xi}$. Note that the orbit of $\xi$ is the discrete set $O_{\xi}$ of infinite sequences of 0's and 1's eventually coinciding with $\xi$, and $\pi_{\xi}$ is a unitary representation of $\mathfrak{S}_{\mathbb{N}}$ in the space $l^{2}\left(O_{\xi}\right)$.

For simplicity, it is convenient to assume that the number of 1's among the first $n$ elements of $\xi$ does not exceed $n / 2$. It is not difficult to see that an arbitrary case can be reduced to this one, but we omit the corresponding technical details.

Denote by $\lambda_{n, k}=(n-k, k)$ the diagram with two rows of lengths $n-k$ and $k$.

Theorem 5. The spectral measure $\mu_{\xi}$ of the cyclic vector $\xi$ in the representation $\pi_{\xi}$ with respect to the Gelfand-Tsetlin algebra is a Markov measure on the space of infinite Young tableaux $T$, and its transition probabilities are given by the following formula. Denote by $m(n)$ the number of 1 's among the first $n$ elements of $\xi$.

$$
\text { If } \xi_{n+1}=0 \text {, then }
$$

$$
\begin{aligned}
& \operatorname{Prob}\left(\lambda_{n, k}, \lambda_{n+1, k}\right)=\frac{n-m(n)-k+1}{n-2 k+1}, \quad \operatorname{Prob}\left(\lambda_{n, k}, \lambda_{n+1, k+1}\right)=\frac{m(n)-k}{n-2 k+1} . \\
& \text { If } \xi_{n+1}=1 \text {, then }
\end{aligned}
$$

$$
\operatorname{Prob}\left(\lambda_{n, k}, \lambda_{n+1, k}\right)=\frac{m(n)-k+1}{n-2 k+1}, \quad \operatorname{Prob}\left(\lambda_{n, k}, \lambda_{n+1, k+1}\right)=\frac{n-m(n)-k}{n-2 k+1} .
$$

Note that all spectral measures of induced representations considered in Theorem 5 are not central (except for the trivial case when one of the sets in the partition is empty).

Example. Let $\xi=0101 \ldots$ Then $m(n)=[n / 2]$, and the formulas for transition probabilities take the following form: 
- if $n$ is odd,

(11)

$$
\operatorname{Prob}\left(\lambda_{n, k}, \lambda_{n+1, k}\right)=\frac{n-2 k+2}{2(n-2 k+1)}, \quad \operatorname{Prob}\left(\lambda_{n, k}, \lambda_{n+1, k+1}\right)=\frac{n-2 k}{2(n-2 k+1)} ;
$$

- if $n$ is even,

$$
\operatorname{Prob}\left(\lambda_{n, k}, \lambda_{n+1, k}\right)=\operatorname{Prob}\left(\lambda_{n, k}, \lambda_{n+1, k+1}\right)=\frac{1}{2} .
$$

It is convenient to rewrite formulas (11), (12) introducing the change of indices $j=n-2 k$. In these terms, a Young tableau is determined by a sequence $\left(j_{1}, j_{2}, \ldots\right)$, where $j_{n}$ takes the values $0,1, \ldots, n$, and the transition probabilities of the measure $\mu_{\xi}$ are equal to

$$
\operatorname{Prob}(j, j+1)=\frac{j+2}{2(j+1)}, \quad \operatorname{Prob}(j, j-1)=\frac{j}{2(j+1)}
$$

at an odd moment of time; and

$$
\operatorname{Prob}(j, j+1)=\operatorname{Prob}(j, j-1)=\frac{1}{2}
$$

at an even moment of time. We see that a random Young tableau governed by the measure $\mu_{\xi}$ is a trajectory of a nonhomogeneous (neither in time nor in space) random walk on $\mathbb{Z}_{+}$. Thus the induced representations of the infinite symmetric group considered in this paper act in spaces of functions over trajectories of natural random walks. Explicit formulas for this action are given by Young's orthogonal form.

5.3. Spectral measure of representations of type $\left(1^{\infty}, \nu\right)$. Consider the representations $I_{\Pi}$ induced from partitions $\Pi$ of type $\left(1^{\infty}, \nu\right)$ with no infinite blocks, infinitely many singletons, and finitely many finite blocks of length greater than one. Here, as above, we denote by $\nu$ the finite Young diagram formed by the lengths of finite blocks of finite multiplicities, and let $|\nu|=n$.

According to Theorem $3, I_{\Pi}$ decomposes into a finite sum of factor representations $\rho_{\mu}$ indexed by Young diagrams $\mu$ with $n$ cells such that $\mu \unrhd \nu$. Let us find the spectral measure $M=M^{\xi}$ of the distinguished cyclic vector $\xi$ with respect to the Gelfand-Tsetlin algebra.

Given $N \geq n$, denote by $\nu_{N}$ the diagram obtained from $\nu$ by adding $N-n$ rows of length 1 . Let $\lambda$ be a Young diagram with $N$ cells. By formula (1), the definition of the spectral measure, and Lemma 2, the cylinder distribution $M_{N}$ of $M$ is given by

$$
M_{N}\left(C_{\lambda}\right)=\left\|P_{\lambda} \xi\right\|^{2}=\frac{\prod \nu_{i} !}{N !} K_{\lambda, \nu_{N}} \operatorname{dim} \lambda .
$$


It is not difficult to see from the definition of the Kostka numbers that

$$
K_{\lambda, \nu_{N}}=\sum_{\mu \unrhd \nu} \operatorname{dim}(\mu, \lambda) K_{\nu, \mu}
$$

where $\operatorname{dim}(\mu, \lambda)$ is the number of paths in the Young graph from $\mu$ to $\lambda$. Therefore, we obtain

$M_{N}\left(C_{\lambda}\right)=\sum_{\mu \unrhd \nu} \frac{\operatorname{dim}(\mu, \lambda) K_{\nu, \mu} \operatorname{dim} \lambda \prod \nu_{i} !}{N !}=\sum_{\mu \unrhd \nu} \frac{K_{\nu, \mu} \operatorname{dim} \mu \prod \nu_{i} !}{n !} \cdot \frac{n ! \operatorname{dim}(\mu, \lambda) \operatorname{dim} \lambda}{\operatorname{dim} \mu \cdot N !}$.

But it is not difficult to check that the second quotient in the right-hand side of the latter formula is exactly the conditional distribution $P\left(\cdot \mid t_{n}=\mu\right)$ of the Plancherel measure $P$ on the space $T$ of infinite Young tableaux $t=\left(t_{1}, t_{2}, \ldots\right)$ (paths in the Young graph) given that at the $n$th level $t$ passes through $\mu$. Note also that the first quotient is the relative dimension of the primary representation corresponding to the diagram $\mu$ in $\operatorname{Ind}_{\mathfrak{S}_{\nu}}^{\mathfrak{S}_{n}}$, i.e., $M_{\text {fin }}(\mu)$, where $M_{\text {fin }}$ is the spectral measure of the distinguished cyclic vector in the representation $\operatorname{Ind}_{\mathfrak{S}_{\nu}}^{\mathfrak{S}_{n}}$ of the finite symmetric group $\mathfrak{S}_{n}$. Thus we obtain the following result.

Proposition 5. The spectral measure $M=M^{\xi}$ of the distinguished cyclic vector $\xi$ in the representation $I_{\Pi}$ is a convex combination of conditional Plancherel measures:

$$
M=\sum_{\mu \unrhd \nu} M_{\mathrm{fin}}(\mu) \cdot P\left(\cdot \mid t_{n}=\mu\right),
$$

where $M_{\mathrm{fin}}(\mu)=\frac{K_{\nu, \mu} \operatorname{dim} \mu \prod \nu_{i} !}{n !}$ is the spectral measure of the distinguished cyclic vector in the representation $\operatorname{Ind}_{\mathfrak{S}_{\nu}}^{\mathfrak{S}_{n}}$ of the finite symmetric group $\mathfrak{S}_{n}$. In particular, it is absolutely continuous with respect to the Plancherel measure $P$ with piecewise constant (cylinder) density

$$
\frac{d M}{d P}(t)=\frac{K_{\nu, \mu}(t) \prod \nu_{i} !}{\operatorname{dim} \mu(t)} \quad \text { if } t \in T \text { passes through the diagram } \mu(t) \text { at level } n .
$$

The Plancherel measure is a central Markov measure on the space of infinite Young diagrams. However, the spectral measure $M$ is not central and is not Markov. However, it is multi-Markov, in the sense that if we "glue" the first $n$ levels in one block, i.e., consider a random Young tableau as a sequence of Young diagrams $\left(\square, \lambda_{n}, \lambda_{n+1}, \ldots\right)$, then it will be a Markov chain.

The following example is the simplest case of a representation of type $\left(1^{\infty}, \nu\right)$, in which the representation is a factor.

Example. A hook with finite hand and infinite leg. If $\nu=(n)$, i.e., the partition consists of one finite block of size $n$ and infinitely many singletons ("a hook with finite hand and infinite leg"), then by Theorem 3(b) the induced representation is a factor, and by Proposition 5 the spectral measure of 
the distinguished cyclic vector is the conditional distribution $P\left(\cdot \mid t_{n}=(n)\right)$ of the Plancherel measure given that at the $n$th level $t$ passes through the one-row diagram $(n)$.

\section{REFERENCES}

[1] M. W. Binder, Irreducible induced representations of ICC-groups, Math. Ann. 294 (1992), $37-47$.

[2] M. W. Binder, On induced representations of discrete groups, Proc. Amer. Math. Soc. 118 (1993), 301-309.

[3] M. W. Binder, Induced factor representations of discrete groups and their types, J. Funct. Anal. 115 (1993), 294-312.

[4] W. Fulton, Young Tableaux. With Applications to Representation Theory and Geometry. Cambridge University Press, Cambridge, 1997.

[5] M. Hamermesh, Group Theory and its Application to Physical Problems, Dover Publ., New York, 1989.

[6] T. Hirai, Some aspects in the theory of representations of discrete groups. I, Proc. Japan Acad. Ser. A Math. Sci. 66 (1990), 315-318.

[7] T. Hirai, Construction of irreducible unitary representations of the infinite symmetric group $S_{\infty}$, J. Math. Kyoto Univ. 31 (1991), 495-541.

[8] I. Macdonald, Symmetric Functions and Hall Polynomials, 2nd edition. Clarendon Press, Oxford, 1995.

[9] G. Mackey, The Theory of Unitary Group Representations, The University of Chicago Press, Chicago-London, 1976.

[10] P. P. Nikitin, A realization of the irreducible representations of $S_{n}$ corresponding to 2-row diagrams in square-free symmetric multilinear forms. Zapiski Nauchn. Semin. POMI 301 (2003), 212-219. English translation: J. Math. Sci. (New York) 129, No. 2, 3796-3799 $(2005)$.

[11] N. Obata, Certain unitary representations of the infinite symmetric group, I, Nagoya Math. J. 105 (1987), 109-119.

[12] N. Obata, Some remarks on induced representations of infinite discrete groups, Math. Ann. 284 (1989), 91-102.

[13] J.-P. Serre, Linear Representations of Finite Groups, Springer-Verlag, New YorkHeidelberg, 1977.

[14] N. V. Tsilevich and A. M. Vershik, On different models of representations of the infinite symmetric group, Adv. Appl. Math. 37 (2006), 526-540.

[15] A. M. Vershik, Strange factor representations of type $\mathrm{II}_{1}$, and pairs of dual dynamical systems, Moscow Math. J. 3, No. 4 (2003), 1141-1157.

[16] A. M. Vershik, A new approach to the representation theory of the symmetric groups, III: Induced representations and the Frobenius-Young correspondence, Moscow Math. J. 6, No. 3 (2006).

[17] A. M. Vershik and S. V. Kerov, Asymptotics of the Plancherel measure of the symmetric group and the limiting form of Young tableaux, Dokl. Akad. Nauk SSSR 233, No. 6, 1024 1027 (1977). English translation: Sov. Math. Dokl. 18, 527-531 (1977).

[18] A. M. Vershik and S. V. Kerov, Locally semisimple algebras. Combinatorial theory and the K-functor. In: Itogi Nauki i Tekhniki, Ser. Sovrem. Probl. Mat., Vol. 26. VINITI, Moscow, 1985, pp. 3-56. English translation: J. Sov. Math. 38, 1701-1733 (1987).

[19] A. M. Vershik and S. V. Kerov, The Grothendieck group of infinite symmetric group and symmetric functions (with the elements of the theory of $\mathrm{K}_{0}$-functor of AF-algebras). In: 
A. M. Vershik and D. P. Zhelobenko (eds.), Representation of Lie Groups and Related Topics, Gordon and Breach Sci. Publ., 1990, pp. 39-118.

[20] A. M. Vershik and A. Yu. Okounkov, A new approach to the representation theory of symmetric groups. II. Zapiski Nauchn. Semin. POMI 307, 57-98 (2004). English translation: J. Math. Sci. (New York) 131, No. 2, 5471-5494 (2005).

[21] A. M. Vershik and N. V. Tsilevich, On the Fourier transform on the infinite symmetric group. Zap. Nauchn. Semin. POMI 325 (2005), 61-82. English translation: J. Math. Sci. (New York) 138, No. 3 (2006), 5663-5673.

[22] A. M. Vershik and N. V. Tsilevich, Markov measures on Young tableaux and induced representations of the infinite symmetric group, Probab. Theory Appl. 51 (2006), 47-63.

[23] H. Weyl, The Theory of Groups and Quantum Mechanics, Dover Pub., New York, 1949.

N. V. Tsilevich

St. Petersburg Department of Steklov Institute of Mathematics

E-mail: natalia@pdmi.ras.ru

A. M. Vershik

St. Petersburg Department of Steklov Institute of Mathematics

E-mail: vershik@pdmi.ras.ru 